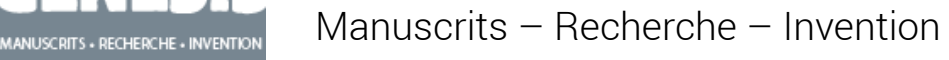

$35 \mid 2012$

Le geste linguistique

\title{
« 53 jours » de Georges Perec : les lieux d'une ruse
}

\section{Maxime Decout}

\section{(2) OpenEdition}

\section{Journals}

Édition électronique

URL : http://journals.openedition.org/genesis/1079

DOI : 10.4000/genesis.1079

ISSN : 2268-1590

\section{Éditeur :}

Presses universitaires de Paris Sorbonne (PUPS), Société internationale de génétique artistique littéraire et scientifique (SIGALES)

\section{Édition imprimée}

Date de publication : 15 novembre 2012

Pagination : 209-219

ISBN : 978-2-84050-869-4

ISSN : 1167-5101

\section{Référence électronique}

Maxime Decout, « « 53 jours » de Georges Perec : les lieux d'une ruse », Genesis [En ligne], 35 | 2012, mis en ligne le 15 novembre 2014, consulté le 19 juin 2020. URL : http://journals.openedition.org/ genesis/1079; DOI : https://doi.org/10.4000/genesis.1079 


\title{
« 53 jours » de Georges Perec : les lieux d'une ruse*
}

\author{
Maxime Decout
}

$\mathrm{L}$

es textes de Georges Perec font assurément partie de ceux dont les avant-textes sont les plus riches et peut-être les plus nécessaires à la compréhension du texte. On y voit une méthode à l'œuvre, une composition, un foisonnement d'exigences, souvent sous la forme de contraintes oulipiennes, mais qui pourtant s'absentent partiellement à la lecture. «Autant je me sers d'une manière stricte ou laxiste de formules et de contraintes au départ, autant au moment du produit achevé, j'essaie que ça ne se voit plus du tout ${ }^{1} »$, affirmait Perec. De ce point de vue, l'avant-texte apparaît comme presque plus vertigineux que le texte. Le regard porté sur les brouillons de Perec indique alors que son écriture est l'antithèse même d'un jaillissement spontané, erratique, dionysiaque. C'est au contraire une organisation rigoureuse et minutieuse qui la commande et voudrait la programmer. Proche en cela des obsessionnels maniaques qu'il met en scène, l'écrivain ne cesse de soumettre ses récits à une planification despotique qui ne laisserait alors plus de place au hasard.

Le dernier roman de Perec, " 53 jours », en témoigne. Interrompu par la mort de l'auteur, ce texte inachevé ne nous est accessible qu'à partir d'une première rédaction et d'un dossier recensant quelques-uns des brouillons sur lesquels travaillait alors l'écrivain. C'est donc dans ce « cabinet d'amateur » où se préparait le texte, où s'interrogeaient l'écriture et le roman, qu'un certain nombre d'éléments se trouvent, sans lesquels la lecture du roman apparaît comme amputée de l'une de ses dimensions. Le projet de Perec était d'écrire un texte qui déconstruirait lui-même sa première partie dans une deuxième partie. Le roman est alors conçu comme une sorte de machine littéraire proposant au lecteur un complexe exercice de lecture et de relecture. Aussi est-ce la spécularité qui intéresse Perec et qui focalise son attention, en travaillant à une intrigue policière plusieurs fois déconstruite et chevillée sur la mise en abyme de pas moins de quatre romans différents, égarant le lecteur dans un jeu confus et vertigineux d'indices et de fausses pistes sans cesse lus et relus.

\section{Notes concernant quelques manuscrits retrouvés dans un cabinet d'amateur}

L'édition proposée par Harry Mathews et Jacques Roubaud $^{2}$ comporte plusieurs éléments : le texte rédigé des onze premiers chapitres de la première partie, ainsi qu'un morceau du douzième chapitre. Viennent ensuite des fragments partiellement rédigés, issus des brouillons, à partir desquels les deux éditeurs ont recomposé une trame narrative fragmentaire : la fin du chapitre 12 et le chapitre 13 de la première partie, puis les chapitres 14 à 28 de la deuxième partie. C'est uniquement pour le chapitre 12 que les fragments sont les plus nombreux. À partir du chapitre 13, ils sont bien plus succincts. De ce fait, c'est principalement la deuxième partie qui apparaît comme lacunaire et pour

\footnotetext{
* Cette étude a été conçue comme la première partie d'un diptyque, dont le second volet, " "53 jours" de Georges Perec : la génétique, mode d'emploi », paraîtra en décembre 2012 dans le numéro 168 de Littérature. L'étude présente est consacrée aux enjeux de la genèse du roman alors que la suivante analyse la manière dont le récit fait de la critique génétique une modalité essentielle mais problématique de la lecture des textes, en la thématisant à travers une enquête policière fondée sur des manuscrits.

1. Cité par Bernard Magné, Perecollages 1981-1988, Toulouse, Presses universitaires du Mirail-Toulouse, coll. "Les cahiers de littératures », 1989 , p. 55.

2. Georges Perec, « 53 jours », éd. Harry Mathews et Jacques Roubaud,
} Paris, Gallimard, coll. « Folio », 1993 [1989]. 
laquelle nous ne disposons que d'un canevas global qui était certainement appelé à des remaniements majeurs. C'est ainsi la deuxième partie, celle qui se propose de relire la première et de la déconstruire, c'est-à-dire celle où se trame l'inachèvement du début, celle qui découd les coutures, qui est restée inachevée : nous n'en avons que l'avant-texte. Impossible donc de savoir quelle forme elle aurait prise, dans quel degré d'achèvement (de surface ?) elle se serait drapée. À ceci, s'ajoute ensuite un aperçu relativement vaste des différents carnets et cahiers préparatoires utilisés par Perec, permettant à la fois d'avoir une vue d'ensemble sur ce qu'aurait peut-être donné la deuxième partie et d'observer la méthode d'élaboration du texte achevé, ou partiellement achevé, de la première partie.

Mais ces carnets ne sont pas datés par Perec, et contiennent en proportion variable des éléments qui se réfèrent à la première ou à la deuxième partie, passant parfois de l'une à l'autre sans vraie distinction. Est-il alors possible d'établir une chronologie approximative ? Le ressassement qui les caractérise semble s'y opposer : Perec mêle des éléments très divers, des listes de noms de personnage, de jeux de mots, des schémas d'intrigue, des allusions, des intertextes, des fragments rédigés... Composites et parfois répétitifs, ces brouillons ne peuvent être considérés comme des versions successives du texte. Pourtant, un certain nombre d'indices permettent de classer ces documents de façon hypothétique.

Il semble d'abord que le cahier Orange soit relativement ancien : il formule effectivement l'hypothèse que le narrateur, à la fin de la deuxième partie, imagine un troisième récit «qui serait l'exégèse contradictoire des 2 premiers » (p. 172). Une idée visiblement abandonnée mais dont on retrouvera la trace dans le texte rédigé à travers le jeu sur les différents romans placés en abyme, dont il n'est fait aucune mention ici alors qu'il est central dans la partie rédigée. De même, dans ce cahier, Perec pense à une première partie écrite à la troisième personne (p. 175), ce qui n'est pas le cas ensuite.

Le carnet Rhodia est, de son côté, plus avancé puisqu'il a trouvé le système d'emboîtement des romans en abyme dont le nom est déjà fixé. En revanche, leur intrigue n'est pas déterminée exactement et commence seulement à se dessiner (p. 194). Le titre des deux parties du texte est quant à lui arrêté, de même que le nom de Salini en tant qu'enquêteur dans la deuxième partie. De plus, le mode de narration de la première partie est choisi et correspond à celui du texte (p. 192). Néanmoins on notera que ce carnet comprend très peu de fragments rédigés, montrant que c'est plus le schéma d'ensemble que le style qui s'élabore. Tout n'est d'ailleurs pas entièrement établi puisque le prénom de Lise est utilisé à la fois pour un personnage de la première partie et pour un autre de la deuxième (p. 187, 190, 192, 193).

Le cahier Bleu, assez bref, contient pour sa part un résumé des grandes lignes de l'intrigue, et semble dans un état d'avancement assez proche du carnet Rhodia, peut-être antérieur puisque l'hypothèse de l'implication de Mme Serval pour le meurtre de son mari y est absente alors qu'elle figure dans le carnet Rhodia (p. 190). Le cahier sur le meuble de chevet retrace lui aussi un synopsis assez proche : la fin de la deuxième partie, comme pour les deux cahiers précédents, n'est pas encore choisie.

Le classeur « 53 jours » est le plus long et celui qui comprend le plus d'éléments rédigés et de schémas. Le narrateur de la première partie s'appelle encore Salini (p. 224). C'est pourtant dans ce cahier que le scénario de la deuxième partie est le plus précis et le plus développé. Il semble avoir trouvé une forme plus avancée. Ce cahier pourrait donc être l'un des plus récents.

Une chose est donc sûre : le cahier le plus primitif est le cahier Orange, alors que Rhodia et « 53 jours » constituent une étape postérieure, plus proche de la rédaction. Cette esquisse de chronologie nous sera utile pour évaluer l'évolution du travail de Perec.

Puisque les fragments rédigés sont toujours relativement brefs, et parfois répétés, sans qu'il y ait de véritable variation stylistique, et que certaines sections présentent une rédaction très incomplète, recourant à des phrases plus ou moins télégraphiques, les brouillons ne nous donnent nullement accès à un quelconque travail stylistique de l'écrivain. D'ailleurs, concernant les sections des cahiers qui portent sur la première partie rédigée, là encore aucun travail sur le style n'est apparent. De ce travail, qui a pourtant permis le passage des notes au texte, nous n'avons donc pas trace, comme si la rédaction et le travail stylis- 
tique s'étaient opérés de façon plus spontanée à partir de la trame narrative et structurelle mise en place dans les brouillons. D'où un constat de taille autorisé par notre effraction dans l'atelier de la création : la « cuisine littéraire » (p. 81) dans « 53 jours », car il s'agit bien d'une sorte de cuisine, est une opération analytique qui ne porte pas en premier lieu sur le style et la rédaction. Pour preuve, de nombreux passages des carnets ont été intégrés tels quels dans la première partie, sans avoir subi de remodelage d'ampleur quant à leur écriture ${ }^{3}$.

Plus qu'un travail stylistique précis, sur ce style que Perec voulait neutre, blanc 4 , les brouillons montrent donc l'essai de différentes structures et clefs. Le travail porte sur la pensée et la conception plus que sur l'esthétique de la phrase. Les changements dans la rédaction, des notes au texte, sont en effet toujours mineurs, affectant quelques adjectifs ou déterminants. C'est bien la fondation d'un système d'allusion et d'encodage qui se donne à lire. Ce sont les listes, combinaisons, chiffrages, et structures, constituant la majeure part du dossier, qui indiquent tous les possibles du jeu que Perec se propose, le foisonnement des effets, des choix qui s'imposent pour la lisibilité et le masquage du sens dans le texte. Trois éléments sont alors récurrents, et semblent pour ainsi dire obsédants pour Perec : le travail sur la diégèse, l'essai d'équilibrage des vingt-huit chapitres entre eux, avec l'établissement de correspondances, et la tentative de faire se correspondre les quatre romans en abyme qui composent le livre.

\section{Tentative d'épuisement d'une intrigue policière}

Dans l'attention soutenue au synopsis du roman, dans la tentative d'envisager plusieurs possibilités de l'intrigue, se donne à lire une véritable maturation de la diégèse. Avec une sorte d'attention quasi maniaque, Perec réécrit à de multiples reprises les mêmes schémas d'intrigue, comme pour les réessayer, les tester, en éprouver l'efficacité et la validité. Il envisage ainsi que, dans la deuxième partie, Serval reçoive le manuscrit, s'interroge et meure « 53 jours » plus tard (Cahier Orange, p. 177). « Oui mais de quoi ? », ajoute-t-il. L'écrivain semble alors parti en quête de tous les possibles pour son histoire, comme si, avant de fixer définitivement le scénario, il fallait au préa- lable tenter d'en épuiser toutes les ressources, pour vérifier que la diégèse choisie fonctionne, soit celle qui corresponde le plus aux effets recherchés. C'est alors la liste que Perec semble priser, plus que le texte rédigé, car elle est à même de recenser le divers et de figurer les étapes narratives :

2e partie

La mort de Serval fut annoncé à la presse le - ..

le célèbre écrivain avait été trouvé dans son bureau

la veille par $\mathrm{X}$

l'autopsie?

parmi les papiers et doc[uments] que l'on trouva sur son bureau

figurait un ms de $\mathrm{X}$ pages (Cahier Orange, p. 178).

Réduite à une sorte de squelette dépouillé de tout ornement romanesque, la diégèse se pense comme enchaînement de séquences, souvent soutenu par une numérotation qui en augmente la lisibilité5. Ce avec quoi le manuscrit semble se battre est assurément le hasard, les licences prises au fil de la plume, les «sauts et gambades ». La rédaction définitive paraît ne devoir se développer qu'à partir d'un carcan fixe. Mais ce cordeau disparaît dans le texte rédigé : la complexité des jeux de miroir, des encodages, des allusions et des mises en abyme, le masquent, donnant au lecteur le sentiment de se perdre dans les méandres d'une intrigue redoutablement complexe et presque immaîtrisée. Il semble ainsi que cette lisibilité préliminaire soit nécessaire à Perec pour mieux contrôler l'égarement, la prolifération et le désordre, qui ne sont alors qu'un effet de surface.

À l'origine de la mise au second plan de la rédaction en tant que telle, ce désir de maîtrise et de structuration du récit ajoute aux listes divers schémas ou organigrammes, comme celui du classeur « 53 jours », assez similaire à celui qui préside à L'Augmentation. Dans cette

3. Bien évidemment, ces passages auraient peut-être été retravaillés ultérieurement par l'écrivain.

4. Voir Georges Perec, W ou le souvenir d'enfance, Paris, Gallimard, coll. « L'Imaginaire », 1993 [1975], p. 63.

5. Voir le cahier Bleu (p. 197-200) où dix-neuf éléments diégétiques numérotés se suivent, l'ensemble du carnet sur le meuble de chevet, p. 207-209, le cahier Orange, p. 182 
figuration spatiale de la pensée, se tient une tentative de mesurer toutes les possibles ramifications de la situation à laquelle Salini est confronté dans la deuxième partie, à partir du manuscrit de Serval (Classeur « 53 jours », p. 238) :

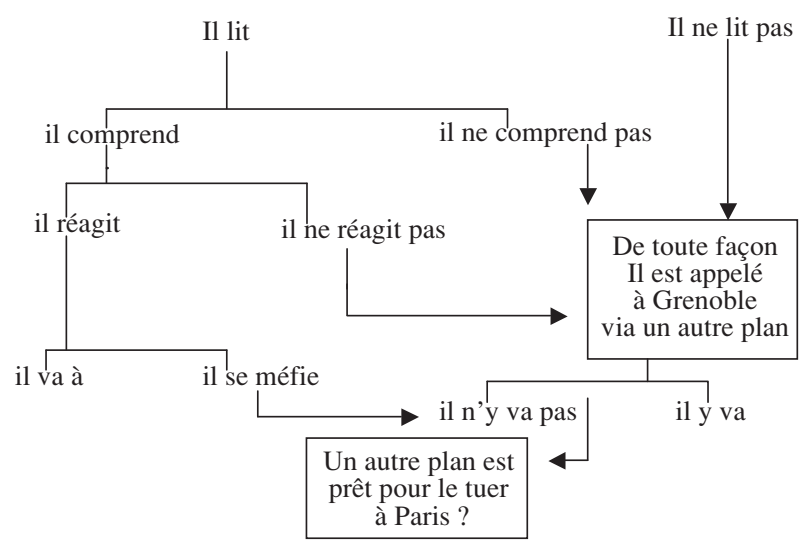

Non destiné à servir de canevas au texte, cet organigramme structure les ramifications de la pensée de l'écrivain. S'il faut tenter de conduire ainsi le personnage vers un but unique selon diverses modalités, c'est bien que ce sont les chemins, les détours et les embranchements, ceux du personnage comme ceux du lecteur, qui importent. Cette attention à l'arborescence est le signe visible d'un foisonnement et d'une actualisation de toutes les combinaisons possibles, donnant à voir la « fascination pour l'exhaustif6 " de Perec que l'on rencontre dans un si grand nombre de textes. Examiner de la sorte, dans les moindres détails, les tenants et les aboutissants d'une situation de départ, témoigne d'une véritable obsession du sens et de son risque d'échappement.

Aussi Perec effectue-t-il souvent de brèves listes d'indices ou d'éléments qui doivent être utilisés dans l'enquête :

Par contre TROUVER QUATRE INDICES FORMANT CHAÎNE
a) fondant un mobile
b) détruisant $\mathrm{t}[\mathrm{ou}] \mathrm{t}$ alibi possible
c) signant le crime (armes, empreintes)
d) donnant témoin (Carnet Rhodia, p. 189).

Éléments de l'enquête

la voiture

le revolver

l'attaché case

« 53 jours » identification de la machine ?

et de la frappe ? (Classeur « 53 jours », p. 244)

N'y aurait-il pas collusion entre les interrogations de l'écrivain, de l'enquêteur et du lecteur? En effet, la méthode d'investigation qui se met en place n'opère pas seulement dans la phase préparatoire mais est reconduite chez le personnage, à travers les interrogations qu'il se pose face à l'enquête et qui seront conservées dans le roman (p. 183, 244) :

horribles hypothèses

p. $14-16$

Mise en scène de S[erval] pour

faire chanter le consul

$\mathrm{OU}$

$$
\begin{aligned}
& \text { scénario inventé } \\
& \text { par le consul pour } \\
& \text { assassiner S[erval] (Classeur « } 53 \text { jours », p. 223) }
\end{aligned}
$$

Pas de faille nulle part peut-être une petite dans l'histoire de Résistance ?

DONC on ne peut que revenir au récit

La 1re cue est: Un R est un M qui se p le 1 d'un C

[...]

Or précisément les faits de résistant de Serval se sont passés en partie à la Chartreuse

d'où l'idée d'un récit codé d'un récit miroir d[on]t le déchiffrement donnerait la clé du mystère (Carnet Rhodia, p. 186).

Ce qui retient l'attention de l'écrivain n'est nullement un adjectif, une tournure syntaxique : ce sont les liens logi-

6. Bernard Magné, Georges Perec, Paris, Nathan université, coll. «Écrivains », 1999, p. 46. 
ques, les rapports de cause à conséquence. Et l'auteur se questionne ici en même temps que son personnage. Comme s'il était lui aussi confronté à une véritable enquête qu'il avait à démêler avant de la rendre inextricable.

Les brouillons dévoilent ainsi une stratégie textuelle calculée qui est destinée à rendre quasi insolubles les questions de l'enquête. Perec essaye différentes combinaisons d'enchaînement et pense avant tout son travail dans l'orbe du questionnement policier qui préside au récit. Cette démarche n'est pas sans conséquence : le principe de causalité reste souverain dans l'enchaînement des actions mais sa prépondérance marquée, sa surdétermination effective, que l'on retrouve dans la partie rédigée, tend à modifier la perception que le lecteur en a, attirant de fait l'attention plus sur les articulations logiques et les zones de bifurcation que sur le reste de l'intrigue, comme si un déplacement d'accent ou de perspective s'était produit. Cette monstration des plis et des replis de la diégèse ressemble à une demande pressante de penser et de réfléchir à l'intrigue elle-même, dans sa dimension structurelle, dans sa syntaxe propre, et ce jusqu'à ne jamais l'accepter sans l'avoir au préalable soupçonnée.

\section{De la difficulté qu'il y a à ranger vingt-huit chapitres}

À cette volonté de construire et de contrôler l'intrigue, s'ajoute aussi la recherche d'une structuration de l'ensemble du livre, et d'abord en fonction des correspondances à établir entre les chapitres de la première et de la deuxième partie. Ce travail résulte de l'objectif initial que s'est fixé Perec : écrire un livre dont la deuxième partie est l'exégèse de la première. Pour cela, c'est la thématique du miroir qui doit saturer l'œuvre, autant dans des références cryptées et ponctuelles, dans le jeu de mise en abyme entre les cinq romans, qu'à l'échelle de la macrostucture du texte. Les essais vont, certainement en raison de l'avancement de la réflexion, de la simple mention (Cahier Orange, p. 175) au tableau qui fait se correspondre en miroir les chapitres des deux parties (Cahier Orange, p. 179) :

$$
\text { 1re partie 2e partie }
$$

\begin{tabular}{|c|c|c|}
\hline & 28 & \\
\hline & 27 & \\
\hline 1 & 26 & \\
\hline 2 & 25 & \\
\hline 3 & 24 & \\
\hline 4 & 23 & \\
\hline 5 & 22 & \\
\hline 6 & 21 & \\
\hline 7 & 20 & \\
\hline 8 & 19 & \\
\hline 9 & 18 & \\
\hline 10 & 17 & \\
\hline 11 & 16 & \\
\hline 12 & 15 & \\
\hline \multirow[t]{3}{*}{13} & 14 & \\
\hline & & $\begin{array}{l}\text { un r[roman] : c'est un } \\
\mathrm{m} \text { [iroir] qu'on } \\
\mathrm{p} \text { [romène] }\end{array}$ \\
\hline & & $\begin{array}{r}\text { le } 1[\text { long]du c[hemin] } \\
\text { S[tendhal] }\end{array}$ \\
\hline
\end{tabular}

On trouve au moins deux autres tableaux similaires, dans le cahier Bleu (p. 201-202) et dans le classeur « 53 jours » (p. 241). Mais dans les deux cas, il n'y a de « rétrocorrespondance », comme ici, entre les chapitres, c'està-dire que le parallèle n'est pas inversé, le chapitre 1 répondant au chapitre 14, le chapitre 2 au chapitre 15. Placée dans le cahier Orange, contenant un état assez primitif du texte, cette progression inversée semble avoir été abandonnée par la suite. Néanmoins c'est la tentative qui importe puisqu'elle fait sens quant au projet du livre. Car, ce que fait apparaître ce tableau, c'est la nécessité de travailler sur la spécularité, à partir de la phrase de Stendhal qui donne son titre à la deuxième partie et qui déclenche la relecture : «Un Roman est un Miroir qui se Promène le Long de la Route ». La symétrie est bien une symétrie inverse, comme un miroir inverse l'image qu'il reflète. De plus, ce schéma isole, comme beaucoup d'autres tentatives d'organisation des chapitres, les chapitres 27 et 28 qui, dans l'état du travail, sont ceux qui donnent la clef du roman : la confession de Patricia, la femme de Serval, à Salini, qui annonce que l'écrivain Georges Perec a été utilisé par elle et son amant pour écrire le texte, en 53 jours, afin d'égarer les enquêteurs 
sur une fausse piste, celle de l'implication de Serval dans une affaire interlope durant la Résistance.

C'est dire l'importance de l'articulation des deux parties. Perec travaille sur les confrontations entre « récit A » et « récit B » (Cahier Rhodia, p. 184-189), qu'il oppose parfois par la dénomination éloquente de « récit A » et « réel ». Il note d'ailleurs que « tout doit être renversé » (p. 187). Au-delà de simples parallèles ou échos, ces renversements s'observent d'abord quant aux situations, échangeant les positions de victime, enquêteur et coupable d'une partie à l'autre. Ces permutations devaient inscrire leur trace dans les plus petits détails, notamment grâce à ces «ANTONYMES sémantiques et logiques » (Carnet Rhodia, p. 189) qu'évoque Perec : l' " histoire de Main Rouge, explication politique vite démentie par $\mathrm{X}$ » pour le récit A se renverse en l'« histoire de résistance confirmée par Salini » pour le récit B, même si l'on sait que cette deuxième histoire sera finalement infirmée, et donc renversée, comme la première. Aussi la jointure des deux parties se conçoit-elle comme un miroir pivotant :

Et tout recommence (à Paris)

sauf que $\underline{t}[\mathrm{ou}] \mathrm{t} \mathrm{p}[\mathrm{ren}] \mathrm{d}$ un nouveau sens (p. 179).

Le sens obsède Perec dans " 53 jours » : celui qui est signification, logique déductive, arborescence rationalisée, mais toujours perturbé, brouillé, attaqué par celui qui est direction changeante, dédale intellectuel, miroir baroque déformant. Le miroir devient alors un « signe », c'est-à-dire un signal quant à la structure inversante du texte, mais aussi un signifiant dont il s'agit de disperser, en l'épuisant, le réseau sémantique qui s'y rattache. C'est ce à quoi sont destinées les listes d'isotopies autour du miroir (Carnet Rhodia, p. 190 ; Classeur « 53 jours », p. 234-235), accompagnées par diverses mentions de jeux consonantiques qui utilisent les deux consonnes du mot, $\mathrm{M}$ et $\mathrm{R}$, ou encore les variations sur les initiales des noms ou verbes dans la phrase de Stendhal : «Un R est un $\mathrm{M}$ qui se P le L d'une R » (Carnet Rhodia, p. 190 ; Classeur « 53 jours », p. 249-257). Le signe du miroir se désigne lui-même dans un processus métatextuel mais cache en même temps ses traces en se cryptant et s'essaimant. Toujours le chemin brouille la signification, toujours le sens égare le sens.

\section{L'art et la manière d'emboîter des récits comme des tables gigognes}

Des difficultés issues de la conception d'une intrigue linéaire, qui se pense pourtant selon une logique de l'arborescence et de la ramification, semble naître une troisième exigence qui occupe une position centrale dans les brouillons : celle de la superposition de plusieurs niveaux de diégèse mis en abyme. Ce troisième aspect essentiel se cheville à la volonté de mettre en pratique, dans l'écriture, la thématique du miroir. L'intrigue d'ensemble est en effet régie par une série d'emboîtements de textes qui servent de fil herméneutique, parfois égarant, au personnage enquêteur mais aussi au lecteur. La première partie fait état d'un narrateur à qui l'on a confié le manuscrit, $L a$ Crypte, d'un écrivain de romans policiers qui a disparu, Serval. Dans ce manuscrit, c'est un dénommé Serval qui, détective, enquête sur une affaire de mise en scène de disparition aux évidentes résonances avec l'affaire qui occupe le narrateur de la première partie. Or Serval personnage recourt, pour mener ses recherches, à un roman policier, Le Juge est l'assassin, dont plusieurs éléments font à nouveau écho aux deux enquêtes précédentes. Mais le narrateur de la première partie découvrira que La Crypte s'inspire de $K$ comme Koala, où nous retrouverons un ensemble d'éléments similaires, bien que toujours légèrement différents. C'est ensuite à la deuxième partie qu'est dévolu le rôle d'un coup de théâtre : la première partie n'a pas d'autonomie en soi, il s'agit du manuscrit de Serval, 53 jours (titre de la première partie), retrouvé dans sa voiture suite à sa disparition. Nous l'apprendrons à la fin, ce texte a en réalité été écrit par Georges Perec pour le compte de la femme de Serval, Patricia, et son amant.

De toute évidence la multiplication des niveaux d'emboîtement égare le lecteur dans un dédale de miroirs, de réalités et d'illusions, où, comme chaque enquêteur, il se perdra. Et, pour ne pas lui aussi se perdre, Perec visualise son dispositif par des tableaux ou des schémas (Carnet Rhodia, p. 193) : 


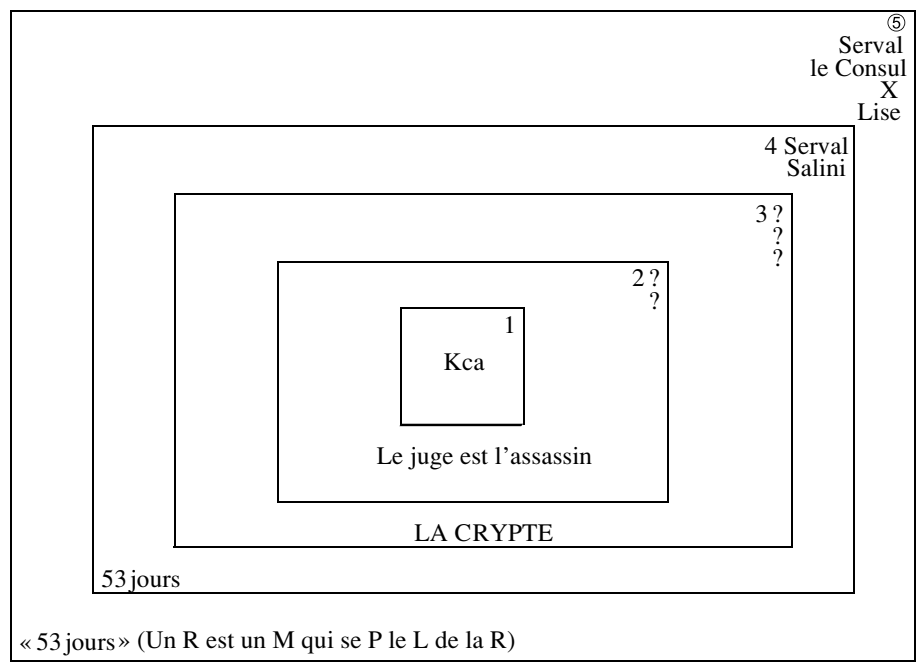

Proche du puzzle, ce dispositif d'imbrication tente de contenir le profus, de résorber le diffus, au sein de cet « espace de type mallarméen dont la structure permet de pousser aux limites la tentative de substituer le construit au fortuit ${ }^{7}$ », qu'est le carré, sorte de figure de pensée idéale chez Perec. La clôture se fait armature comme ligature. Nulle rencontre hasardeuse entre les textes en abyme mais une sorte de mariage concerté qui est pensé dans les moindres détails. Aussi trouve-t-on plusieurs tâtonnements quant à l'intrigue des romans emboîtés afin de faire jouer à plein régime le principe de symétrie et d'allusions (Carnet Rhodia, p. 194). Perec travaille alors sur des positions actantielles en miroir, celles de la victime, de l'assassin et de l'enquêteur (Pages pliées dans le classeur « 53 jours », p. 289) :

\begin{tabular}{|c|c|c|c|}
\hline & $2^{\mathrm{e}}$ récit & $3^{\mathrm{e}}$ récit & $1^{\mathrm{er}}$ récit \\
\hline Consul & Détective & Coupable & Victime \\
\hline Serval & ${ }^{\downarrow}$ Coupable ${ }^{\uparrow}$ & Victime & Détective \\
\hline Xavier & Victime & Détective & Coupable \\
\hline
\end{tabular}

Une véritable circulation se met en place entre les textes en abyme, circulation que l'on retrouve en effet dans le texte, mais de façon beaucoup moins maitrisée que ne le voulaient les brouillons. Le schéma, qui n'utilise ici que trois récits, certainement ceux de la première partie, ne parvient pas à rendre entièrement compte de la complexité de l'opération qui se déroule finalement dans le texte rédigé.

Si cet ajustement arithmétique échoue c'est parce que le schéma néglige en réalité les effets de transfert d'une position actantielle à l'autre au sein même de chaque roman. Les intrigues en abyme reposent en effet sur un système de permutation interne où un même personnage peut être, par exemple, fausse victime et coupable. Le sens que le texte construit est donc encore perturbé par des modifications de direction et par l'effet miroir (Classeur « 53 jours », p. 237) :

\begin{tabular}{ccc|cc} 
& Assassin réel & $\begin{array}{c}\text { Victime } \\
\text { réelle }\end{array}$ & $\begin{array}{c}\text { Victime } \\
\text { Miroir }\end{array}$ & $\begin{array}{c}\text { Assassin } \\
\text { miroir }\end{array}$ \\
JUGE & Vidornaught & Angèle & & Fly \\
CRYPTE & Rouard & Vichard & Rouard & Vichard \\
$53 \mathrm{~J}$ & Serval & Consul & Serval & Narrateur \\
«53 J » & $\begin{array}{c}\text { Mme Serval } \\
+ \text { consul } \\
\text { (amant) }\end{array}$ & $\begin{array}{c}\text { Serval } \\
\text { (héritage) }\end{array}$ & $\begin{array}{c}\text { Serval } \\
\text { (Résistance) }\end{array}$ & $\begin{array}{c}\text { « Consul } \\
\text { (réseau) } \\
\text { Chabert }\end{array}$
\end{tabular}

Passant du rêve de clôture à un essai de nomenclature, ce tableau met en exergue la manière dont un personnage peut occuper une position réelle et une position «miroir», c'est-à-dire une position qu'il usurpe de son plein gré ou contre son gré à cause d'une ruse. Il est pourtant évident que cet état des choses ne correspond pas exactement à la version rédigée puisque Vidornaught par exemple est un personnage de K comme Koala et que la situation décrite dans La Crypte est ici simplifiée. L'harmonie, suscitée au moment de la première réflexion sur la spécularité, grâce à l'infrastructure des carrés imbriqués, a donc laissé la place à un inquiétant foisonnement. La profusion met en échec la capture dans la clôture. Les cases ne peuvent être étanches. Parti d'un équilibre défini et maîtrisé, Perec aboutit à une sorte d'impossibilité.

7. Bernard Magné, op. cit., p. 78. 
En effet, un tableau plus exact de la situation finalement choisie dans le texte dont nous disposons pourrait être proposé, tenant compte de ceux établis par Perec dans ses brouillons :

\begin{tabular}{c|c|c|c|c|c} 
& Enquêteur & $\begin{array}{c}\text { Coupable } \\
\text { réel }\end{array}$ & $\begin{array}{c}\text { Victime } \\
\text { réelle }\end{array}$ & $\begin{array}{c}\text { Victime } \\
\text { miroir }\end{array}$ & $\begin{array}{c}\text { Coupable } \\
\text { miroir }\end{array}$ \\
KcK & aucun & KGB & Legros & Blanes & Vidornaught \\
JUGE & aucun & Tissier & Angèle & Tissier & Fly \\
La Crypte 1 & Blackstone & Rouard & aucune & Rouard & Vichard \\
La Crypte 2 & Serval & Vichard & Rouard & aucune & Vichard \\
53 J & Narrateur & Serval & Consul & Serval & Narrateur \\
« 53 J $»$ & Salini & $\begin{array}{c}\text { Femme } \\
\text { de Serval }\end{array}$ & Serval & $\begin{array}{c}\text { Serval } \\
\text { résistant }\end{array}$ & Chabert \\
& & Consul & & &
\end{tabular}

Quelques paramètres supplémentaires apparaissent : le statut de l'enquêteur, le fait que le coupable n'est pas nécessairement un assassin puisqu'il n'y a pas toujours meurtre, et le fait que l'intrigue de La Crypte présente en réalité un double dénouement sans résoudre l'enquête. En effet, Serval enquêteur propose deux hypothèses différentes pour expliquer l'affaire (p. 61-62) : soit Rouard est le coupable qui a mis en scène son meurtre pour faire accuser Vichard (noté ici La Crypte 1), soit Vichard s'est arrangé pour être accusé puis innocenté du meurtre de Rouard qu'il a réellement commis (La Crypte 2). Les positions actantielles diffèrent évidemment si l'on envisage l'une ou l'autre hypothèse.

Pourtant, là encore, l'armature laisse voir ses limites. Par exemple, la dénomination de « victime miroir » ne désigne pas, dans $K$ comme Koala, une fausse victime que l'on fait passer pour telle, mais une victime supposée qui se révélera avoir survécu. La congruence rêvée est impossible. Même plus, elle serait peut-être indésirable. On constate en effet que les correspondances exactes entre les cinq textes avortent certes parce que la complexité de la composition réticulée est trop grande mais aussi parce que
Perec les fait avorter. Alors même que celui-ci semble avoir mesuré et interrogé les récits en abyme dans ses brouillons, une certaine désinvolture vient marquer le traitement réservé à leur intrigue dans le texte. La conclusion ambiguë de Serval quant à la résolution de l'affaire de La Crypte, pour le moins inhabituelle, liquide l'édifice de l'enquête, patiemment élaboré, soulignant le peu d'intérêt qu'elle recèle en elle-même et déroutant le lecteur, comme le narrateur, plus qu'elle n'apporte un réel soutien (p. 61-62). Il en va de même pour l'intrigue de $K$ comme Koala qui épouse un schéma narratif plus éloigné des autres textes, et qui est résumée avec ironie par un narrateur lassé et soulignant à l'envi les complications gratuites d'un tel récit (p. 88-92). Dans une moindre mesure, l'impossible congruence vient aussi du fait que Le Juge est l'assassin et $K$ comme Koala ne comportent pas d'enquêteur alors que La Crypte en présente trois, mais dont les deux premiers, Derville et Blackstone, ne sont là que pour servir d'allusion codée à Stendhal. Le méticuleux lecteur, s'étant glissé avec soin dans la peau de l'inspecteur, cherchant à établir des parallèles minutieux entre les textes, comme le fait le narrateur dans la première partie, se trouve devant un problème inextricable et s'enfonce peu à peu dans des complications plus redoutables que celles de départ. La mise en abyme est le lieu d'une ruse qui affirme que le texte est un dédale même s'il peut servir de clef. Testant le degré de vraisemblance de ses intrigues ainsi que leurs possibles combinaisons, l'écrivain construit sa propre fascination et celle du lecteur. D'où cette sorte de pulsation singulière qui fait passer, dans le texte, de l'acquiescement à la distance ironique quant à la lisibilité des intrigues en abyme.

De cette profonde stratification de l'intrigue, de l'attention aux ramifications envisagées pour la diégèse des deux parties, comme du travail d'équilibrage des chapitres, apparaît donc un désir de cerner toutes les branches d'une histoire sans pouvoir toutes les conserver dans le texte. La structure d'emboîtement des romans est alors un viatique pour réintroduire le foisonnement des possibles. Une seule intrigue, dans la structure linéaire et mimétique qui préside à « 53 jours », et ce contrairement à un texte délibérément détaché de tout réalisme et de toute logique diachronique comme celui de L'Augmentation, ne peut contenir l'ensemble des hypothèses plausibles qui permettraient de 
ramifier l'histoire. Le modèle de l'arborescence, que les brouillons esquissent à plusieurs reprises, est donc inadéquat, hormis lorsque c'est le personnage qui considère les événements passés en essayant d'en reconstruire les éventuels enchaînements. Mais, pour les événements que vivent les personnages, une telle structuration est hors de propos. Aussi c'est bien une démultiplication hors du commun de l'intrigue qu'autorise la mise en abyme à cinq niveaux. Comme si Perec travaillait son récit autant selon un axe horizontal que selon un axe vertical, comme si le linéaire, grâce au spéculaire, était complété par le tabulaire. Il s'agit donc de brancher sur une matrice linéaire une syntaxe narrative qui multiplie les entrecroisements et les juxtapositions. Perec, dans sa « volonté d'épuisement combinatoire ${ }^{8}$ », a alors besoin du miroir de cinq diégèses, jamais tout à fait la même ni tout à fait une autre, pour épuiser la richesse d'un même scénario originel, pour conduire à l'exhaustif.

\section{De l'usage de la contrainte}

Le geste génétique dans « 53 jours » est donc un effort de rationalisation et de structuration, inscrit de toute évidence dans le sillage de l'appartenance de Perec à l'Oulipo. C'est pourquoi la répartition des romans en abyme s'essaye aussi en regard de l'importance quantitative à leur accorder, par le biais d'une contrainte. Pour ce faire, Perec tente à plusieurs reprises de les ordonner selon une suite de Fibonacci (Classeur « 53 jours », p. 236-237, 240), déjà utilisée par l'écrivain dans certains poèmes. Celle-ci repose sur un principe numérique simple : chaque nombre est la somme des deux précédents. Le but de Perec est d'obtenir un total de 53, et il dresse alors la liste de toutes les additions possibles pour arriver à ce résultat (p. 240). Pourtant il note « essayer de s'y tenir mais pas trop grave si ça déborde it's just a detail » (p. 237). On trouve d'ailleurs la mention occasionnelle du bi-carré latin d'ordre trois (Pages pliées dans le classeur « 53 jours », p. 290), structure qui, d'ordre 10, avait été utilisée pour $L a$ Vie mode d'emploi, mais sans qu'elle aboutisse ici vraiment. De même, la liste des vingt-huit contraintes établie par Perec (Pages pliées dans le classeur « 53 jours », p. 290), en en fixant arbitrairement une par chapitre, essayant de réutiliser la féconde méthode qui avait permis l'élaboration de La Vie mode d' emploi, montre bien à quel point ce système n'opère pas ici : cette liste est unique, Perec n'y revenant nulle part ailleurs dans ses brouillons. Elle n'a pas été suivie, du moins pour l'élaboration de la partie rédigée, et elle se trouve dans une partie relativement ancienne des brouillons. La contrainte oulipienne, au sens le plus strict, ne semble alors d'aucun secours.

L'essai de suites de Fibonacci, de répartition des romans, de différentes versions de l'intrigue, de correspondances entre les chapitres montrent ainsi que la méthode utilisée par Perec est relativement différente de celle de La Vie mode d' emploi, où les échafaudages et les structures cachées sont essentiels. Ceux-ci ont d'ailleurs été rendus accessibles par la publication du « Cahier des charges 9 ». Avec « 53 jours », ces deux textes sont donc ceux pour lesquels l'on dispose d'un dossier génétique publié qui met l'accent sur le travail structurel plus que scriptural, sur la syntaxe du récit plus que sur celle de la phrase. Mais, contrairement aux « romans » de Perec, « 53 jours » ne résulte pas d'un ensemble de contraintes préexistantes, fixées à l'avance dans un « cahier des charges » mais bien de tâtonnements divers, où chaque procédé essayé n'est pas une contrainte de départ mais un moyen de structurer le texte et d'y inscrire, de la manière la plus variée possible, la réflexion qu'il propose sur la spécularité. " 53 jours », comme $W$ ou les romans pré-oulipiens, ne résulte donc pas de contraintes dures. Perec déclarait : «presque aucun [de mes livres] [...] ne se fait sans que j'aie recours à telle ou telle contrainte ou structure oulipienne, ne serait-ce qu'à titre symbolique et sans que ladite structure ou contrainte me contraigne en quoi que ce soit 10 ». « 53 jours » relèverait peut-être de cet usage « symbolique » de la contrainte. Car chaque procédé n'est somme toute que peu contraignant, il ne s'agit pas de carcans établis d'avance d'où l'écriture naîtra mais bien de procédés essayés pour accompagner cette écriture.

\section{Bernard Magné, op. cit., p. 47.}

9. Georges Perec, Cahier des charges de La Vie mode d'emploi, éd. Hans Hartje, Bernard Magné et Jacques Neefs, Paris, CNRS Éditions/Zulma, 1993.

10. Georges Perec, Penser/Classer, Paris, Éditions du Seuil, coll. « La librairie du XxI ${ }^{\mathrm{e}}$ siècle », 2003 [1985], p. 10-11. 
De ce fait, alors même que « 53 jours » ne résulte pas d'une contrainte dure de départ, contrairement à La Disparition, emblème de l'œuvre oulipienne, les deux textes peuvent être rapprochés. Dans La Disparition, c'est la contrainte d'écriture lipogrammatique qui est centrale, à l'échelle microtextuelle, et qui est seulement ensuite relayée au niveau du dispositif structurel (avec la suppression d'un chapitre, d'une partie) et thématique (avec les disparitions en chaîne). Mais cette structure n'est pas au premier chef déterminée par la contrainte. Il semble ainsi que la structure d'ensemble de " 53 jours », contrairement à celle de La Vie mode d' emploi mais semblable à celle de La Disparition, ne réponde pas directement à une contrainte mais soit la résultante de l'enjeu central que Perec a placé à l'origine de son texte. Les procédés utilisés ne sont donc pas là par décision arbitraire qui seule les justifie mais ils trouvent leur justification dans leur efficacité, dans ce qu'ils produisent et non dans l'acte seul de produire indépendamment du résultat, comme c'est le cas dans la liste des éléments à inclure dans chaque chapitre de La Vie mode d'emploi, tel un événement survenu pendant l'écriture, une posture, une citation qui ne sont pas déterminés en fonction de leur contenu final mais par décision antérieure à leur écriture. C'est bien ce que montrent les divers essais de Perec dans les avant-texes de " 53 jours », ainsi que les échecs et abandons de certains procédés qui n'ont pas permis de réaliser l'effet escompté. Au départ du livre, plus qu'une contrainte, se tiendrait une volonté première, qui serait l'idée séminale d'où l'ensemble de l'écriture aurait germé : écrire un livre miroir. De ce choix initial découlent trois grands principes, à partir desquels vont s'essayer l'ensemble des processus d'écriture : écrire un texte où la deuxième partie est la déconstruction de la première, se référer à Stendhal et emboîter plusieurs textes en abyme. Ce sont ces trois axes de départ d'où découle l'ensemble des recherches d'anagrammes, d'allusions, de correspondances... Chaque utilisation d'un principe formel est ainsi destinée à conforter le sens. Les citations ou allusions, à divers auteurs, dont Stendhal, ne suivent apparemment aucune règle mais viennent s'intégrer librement dans le texte, à la manière dont Perec procédait dès ses premiers romans, dans Les Choses ou Un homme qui dort, alors que la citation et l'allusion sont majoritairement programmées dans La Vie mode d'emploi. Il semble ainsi que le hasard et la liberté d'écriture refluent finalement au-delà des carcans rigides avec lesquels l'écrivain essaye de borner et de contrôler son texte dans les brouillons. Le rêve de la clôture du carré a été dépassé.

L'étude des brouillons de " 53 jours » permet ainsi de mesurer l'évolution progressive des méthodes de travail employées par Perec pour ses romans. Dans son dernier texte, l'écrivain se montre principalement préoccupé de questions d'organisation d'ensemble, affrontant une profusion difficilement maîtrisable. Dans ce travail sur la dispositio, les états différents des brouillons montrent la volonté de conserver activement des virtualités inaccomplies au sein du roman. Cultivant donc jusqu'à sa dernière œuvre une poétique de la bigarrure et de la prolifération, qu'il s'agit toujours d'étager sans les simplifier, Perec prolonge la brocante romanesque de La Vie mode d' $\mathrm{em}$ ploi mais cette fois sans fragmenter l'intrigue. Ces deux romans de Perec relèvent ainsi d'une même logique : celle du puzzle. Conjoignant la structuration à la fragmentation, les deux ouvrages mettent en balance la clôture et l'aperture. Car si la structure se rêve clôture sans jamais réussir pleinement, elle demeure aussi ce qui fait lien entre des éléments disparates. C'est pourquoi texte et avant-texte de " 53 jours », s'ils sont les lieux d'une ruse et d'un jeu avec le lecteur, sont aussi essentiellement les lieux d'une ruse avec l'indépassable menace qui plane toujours derrière les constructions savantes, les procédés oulipiens, la légèreté ou l'humour chez Perec : celle du vide et du blanc. 
Maxime Decout, agrégé et docteur en Lettres modernes, maître de conférences à l'université Lille III, est l'auteur d'Albert Cohen : les fictions de la judéité (Paris, Classiques-Garnier, 2011) et a dirigé le numéro d'Europe consacré en janvier 2012 à Georges Perec. Il travaille notamment sur les rapports entre littérature et judéité et a publié plusieurs articles sur Albert Cohen (Poétique, Les Temps modernes, Études littéraires, Genesis...), Georges Perec (Europe, Poétique, Roman 20-50), Hélène Cixous (Critique) et Patrick Modiano (Littérature).

maximedecout@yahoo.fr

Résumés

\section{« 53 jours » de Georges Perec : les lieux d'une ruse}

Le projet de Perec avec « 53 jours » était d'écrire un texte qui déconstruirait lui-même sa première partie dans une seconde partie. Aussi est-ce la spécularité qui focalise le travail de Perec dans les brouillons, autour d'une intrigue policière plusieurs fois déconstruite et chevillée sur la mise en abyme de quatre romans différents. Ce sont les listes, combinaisons, chiffrages, schémas et structures qui retiennent le travail de l'écrivain dans une volonté de mise en ordre et d'épuisement de tous les possibles. Trois éléments sont alors récurrents : le travail sur une diégèse ramifiée, l'essai d'équilibrage des vingt-huit chapitres entre eux, et la tentative de faire se correspondre les quatre romans en abyme qui composent le livre. Confronté à une volonté de mettre en ordre, le système mis en place par Perec est pourtant toujours débordé par un certain désordre. Il semble alors que " 53 jours » ne résulte pas de contraintes préalables à l'écriture mais que les dispositifs mis en place soient essayés et trouvés au cours de la rédaction afin de répondre à l'enjeu de départ que s'était fixé Perec.

Perec, with "53 jours", wanted to write a book in which he would deconstruct its first part in a second one. As a result the manuscript works on the mise en abyme of four detective novels based on a deconstructed and articulated plot. Perec uses lists, combinations, encodings and sketches to organize the profusion of possibilities. There are three recurrent elements: the work on a branched story, the attempt to balance the twenty eight chapters, and the search for correspondences between the novels put in abyme. But, faced with the desire for order, the system sought by Perec always yields to disorder. Apparently "53 jours" is not the result of previous constraints but of devices that Perec sought and found in the course of writing to attain the goal he had set for himself.

Mit „53 jours “ verfolgte Perec den Plan, einen Text zu schreiben, der selbst seinen ersten Teil in einem zweiten Teil dekonstruiert. Diese Vision verfolgt Perecs Arbeit auch in seinen Entwürfen rund um eine Kriminalintrige, die anhand der „Mise en abyme“ vier verschiedener Romane mehrfach dekonstruiert und neu zusammengefügt wird. Listen, Kombinationen, Berechnungen, Schemata und Strukturen halten die Arbeit des Schriftstellers in seinem Willen, alle Möglichkeiten und Anordnungen auszuschöpfen, fest. Drei Elemente wiederholen sich dabei: die Arbeit an einer verzweigten Diegenese, der Versuch eines Gleichgewichts zwischen den 28 Kapiteln und die Absicht, die vier Romane „en abyme“, aus denen das Buch besteht, in Übereinstimmung zu bringen. Doch entgegen seines Willens nach Ordnung endet das von Perec entwickelte System immer wieder in einem gewissen Durcheinander. Es scheint deshalb, dass ,,53 jours “ nicht aus dem Schreibprozess vorangegangenen Zwängen heraus entstanden ist, sondern dass die eingesetzten Mechanismen im Verlauf des Verfassens ausprobiert und gefunden wurden, mit dem Ziel, eine Antwort auf die von Perec zu Beginn festgelegte Herausforderung zu geben.
E1 proyecto de Perec, con "53 jours", era escribir un texto, cuya segunda parte deconstruyera la primera. Por ello, la especularidad focaliza el trabajo de Perec en los borradores, alrededor de una intriga policial varias veces deconstruida y sujeta a la mise en abyme de cuatro novelas diferentes. Son las listas, combinaciones, cuantificaciones, esquemas y estructuras que acaparan el trabajo del escritor buscando poner en orden y agotar todas las posibilidades. De allí que tres elementos sean recurrentes: el trabajo sobre una diégesis ramificada, el esfuerzo por reequilibrar entre ellos los veintiocho capítulos y la tentativa de establecer una correspondencia entre las cuatro novelas en abyme que componen el libro. Confrontado a una voluntad de poner orden, el sistema instaurado por Perec sucumbe sin embargo ante un cierto desorden. Resulta evidente entonces que "53 jours" no es el resultado de disposiciones previas a la escritura sino que los dispositivos son experimentados y descubiertos en el momento de la redacción como respuesta a los objetivos iniciales que se había finado Perec.

Com "53 jours", Perec projectava escrever um texto que desconstruiria a primeira parte, dando como resultado a segunda. Daí a visão especular que enfoca o trabalho de Perec nos rascunhos, em redor de uma intriga policial várias vezes desconstruida e alicerçada na "mise en abyme" de quatro romances diferentes. Listas, combinações, codificações e esquemas são estruturas que contêm o trabalho do escritor numa vontade de organização e de esgotamento dos recursos possíveis. Três elementos são recorrentes: o trabalho sobre uma diegese ramificada, a tentativa de equilíbrio entre os 28 capítulos e a busca de correspondências entre os quatro romances "en abyme" que formam o livro. Confrontado com o desejo de organizar, o sistema montado por Perec acaba, no entanto, ultrapassado por certa desordem. Parece assim que " 53 jours" não resulta de normas estabelecidas previamente à escrita, mas que os dispositivos instalados foram achados e experimentados durante a redacção como respostas ao desafio de partida que Perec se fixou.

Il progetto di Perec, con "53 jours", era di scrivere un testo che decostruisse esso stesso la sua prima parte in una seconda parte. È la specularità che focalizza il lavoro di Perec nei manoscritti, attorno a un intrigo poliziesco smontato e ricostruito più volte sulla myse en abyme di quattro diversi romanzi. Liste, combinazioni, cifrature, schemi e strutture manifestano la volontà dello scrittore di mantenere l'ordine e di esaurire tutti i possibili. Tre elementi ricorrono: il lavoro su una storia ramificata, la costruzione di un equilibrio dei ventotto capitoli tra loro, e il tentativo di fare corrispondere i quattro romanzi en abyme tra loro. Stimolato da questa volontà, il sistema organizzato da Perec è tuttavia perturbato da un certo disordine. Sembra allora che "53 jours" non derivi da limiti prestabiliti ma che Perec misuri e crei i meccanismi del testo nel corso della redazione, al fine di rispondere alla sfida che si era posto fin dall'inizio. 